\title{
Physical Properties of the B and Be Star Populations of $h$ and $\chi$ Persei
}

\author{
Amber N. Marsh Boyer ${ }^{1}$, M. Virginia McSwain ${ }^{1,2}$, Christina Aragona ${ }^{1}$, \\ and Benjamin Ou-Yang ${ }^{3}$ \\ Department of Physics, Lehigh University, 16 Memorial Drive East, Bethlehem, PA 18015 \\ anm506@lehigh.edu, mcswain@lehigh.edu, cha206@lehigh.edu, ouyang@chara.gsu.edu
}

\begin{abstract}
We present a study of the B and Be star populations of the Double Cluster $h$ and $\chi$ Persei. Blue optical spectroscopy is used to first measure projected rotational velocity, $V \sin i$, effective surface temperature, $T_{\text {eff }}$, and surface gravity, $\log g$, for B-type sample stars, while available Strömgren photometry is used to calculate $T_{\text {eff }}$ and $\log g$ for the Be stars showing emission. In our sample of 104 objects for which we measured these stellar parameters, 28 are known or proposed Be stars. Of these Be stars, 22 show evidence of emission at the times of our observations, and furthermore, we find evidence in our data and the literature for at least 8 transient Be stars in the clusters. We find that the Be stars are not rotating near their critical velocity, contrary to the results of studies of similar open clusters. We compare the results of our analysis with other previous studies, and find that the cluster members are more evolved than found by Huang \& Gies but still retain much of their initial rotational angular momentum.
\end{abstract}

Subject headings: open clusters and associations: individual (NGC 869, NGC 884) - stars: emission-line, $\mathrm{Be}$

\section{Introduction}

NGC 869 and NGC 884 ( $h$ and $\chi$ Persei, respectively) are a well known double open cluster, visible in the Northern hemisphere, and have been the focus of many studies over the years. The early 1900 's saw a number of studies attempting to determine cluster membership, positions, and radial velocities (Messow 1913; Adams \& VanMaanen 1913; Hertzsprung 1922). By the 1960's more extensive studies, such as that of Slettebak (1968), were being conducted to determine spectral types for the

\footnotetext{
${ }^{1}$ Visiting Astronomer, Kitt Peak National Observatory, National Optical Astronomy Observatory, which is operated by the Association of Universities for Research in Astronomy (AURA) under cooperative agreement with the National Science Foundation.

${ }^{2}$ The WIYN Observatory is a joint facility of the University of Wisconsin-Madison, Indiana University, Yale University, and the National Optical Astronomy Observatory.

${ }^{3}$ Current address: Department of Physics and Astronomy, Georgia State University, P.O. Box 4106, Atlanta, GA 30302
}

cluster constituents. More recently an extensive study has been conducted by Currie et al. (2010) in which they investigated the general properties and membership of the clusters. Their results, in agreement with those of Bragg \& Kenyon (2005) and Slesnick et al. (2002), find that the clusters are incredibly similar, having common ages of $\sim 13-14$ Myrs, distance moduli $\mathrm{dM}=11.8-11.85(\sim 2,200$ $\mathrm{pc})$, and reddenings of $\mathrm{E}(B-V) \sim 0.52-0.55$. They also estimate a total mass of at least $20,000 \mathrm{M}_{\odot}$ for the clusters.

One of the prominent motivations for our study is that these young open clusters are rich in Be stars. As early as the 1920's, observational studies conducted by Trumpler (1926) and others noted the presence of emission in the hydrogen lines of many of the brightest B-type cluster members. Modern studies of the cluster have shown that upwards of $30 \%$ of the brightest B-type stars are known to be Be stars (Keller et al. 2001). In a study conducted with Spitzer, Currie et al. (2008) investigated the 
lower mass stellar population for mid-infrared excesses due to the presence of protoplanetary disks. They also identified 57 Be stars and candidates exhibiting excess emission at $24 \mu \mathrm{m}$, which helped to motivate our study to follow up these candidates and confirm their Be nature. Of their stars, 21 had previously been identified as showing emission, and 20 of their stars are included in the present study.

The modern working definition of a Be star is given as "a non-supergiant B star whose spectrum has or had at some time, one or more Balmer lines in emission" (Porter \& Rivinius 2003). While this general definition also encompasses objects such as the well known Algol binary systems and Herbig Ae/Be stars, classical Be stars are further delineated as having circumstellar line emission formed in an optically thin equatorial disk, low-order line profile variations, and rapid rotation (Porter \& Rivinius 2003). These Be disks are comprised of warm gaseous material ejected from the stellar surface during outburst events. The gas is then pulled into a gravitationally bound orbit about the stellar equator.

It is well established that as a population, $\mathrm{Be}$ stars rotate faster than than their non-emission, Btype counterparts. Precisely why this is the case, however, is still debated. There are three primary theories as to why Be stars are rapid rotators: they may have been born as rapid rotators, spun up by mass transfer in a close binary system, or spun up during the main sequence evolution of B-type stars. The observed rotation rates of Be stars are $\geq 60-80 \%$ of their critical velocity (McSwain et al. 2008), at which point the gravitational and centrifugal forces are balanced, although recent results suggest that this threshold may be mass dependent (Huang et al. 2010). The main sequence lifetimes of these objects are likely extended as a direct result of their rapid rotation, as this fosters rotational mixing of their stellar interiors and replenishes their hydrogen cores (Mevnet \& Maeder 2000). However, rapid rotation alone is not enough to spur the photospheric material of these stars to form the disk structures they host. It is likely that other weaker processes, such as non-radial pulsations (NRPs), are needed to provide the additional angular momentum necessary for this material to leave the stellar surface (Rivinius et al. 2001; Porter \& Rivinius 2003; McSwain et al. 2008; Cranmer 2009). A growing number of Be stars have been identified to exhibit NRPs (see Rivinius et al. 2003; Emilio et al. 2010).
B-type and Be stars have become important targets for asteroseismology. In hot stars, different pulsation frequencies provide information about different layers of the stellar interior. The information gleaned from studies targeting B stars with NRPs is being used to improve current stellar structure and stellar evolution models. Doing so, however, requires accurately determined stellar surface parameters in order to set appropriate boundary conditions for these models. Accurate measures of effective temperature and surface gravity are particularly essential to determining stellar radii for these models, as well as for determining stellar ages and evolutionary spin-down. Many B-type and Be stars in $h$ and $\chi$ Per have been found to host NRPs, and currently there are on-going campaigns to observe and characterize the nature of the variable pulsations found in these stars Krzesiński \& Pigulski 1997; Krzesiński et al. 1999; (Saesen et al. 2010). The interest in the pulsating stars of $h$ and $\chi$ Per necessitates improved measurements of stellar parameters for the cluster.

There is an on-going debate in the massive star community regarding the evolution of angular momentum of B-type stars. With their abundance of B-type stars, $h$ and $\chi$ Per are two of the many stellar clusters at the center of this debate. Strom et al. (2005) find that the present-day rotation rates of these stars are set by environmental characteristics of the natal clouds in which they formed, with little change over the main sequence stellar lifetime. The work of Huang \& Gies (2006a) and Huang et al. (2010), however, indicates that the observed rotation rates of B-type stars are due less to the initial birth-line rotational rates of the stars and more to evolutionary spin-down or mass transfer in binaries. Both studies also observe that B-type stars in clusters have, on average, significantly higher rotation rates than field B-type stars. Resolving the angular momentum problem also requires a new examination of the stellar parameters and evolutionary states.

In this work we present stellar properties determined from blue optical spectroscopy of a sample of B and Be stars in the clusters. Section 2 provides details of spectroscopic observations taken with various instruments. In Section 3 we discuss our methods for determining $V \sin i, T_{\text {eff }}$, and $\log g$ for both the B and Be star populations. We feel that in order to study Be stars relative to their 
non-emission peers it is important to apply a uniform technique for B and Be star analysis (in as much as possible). With this homogenous and independently determined dataset we will be able to further investigate the nature of the clusters' Be star population. Section 4 quantitatively compares our results with those of Huang \& Gies (2006a) and Strom et al. (2005), who use different methods to determine the same physical parameters. We discuss the impact of our disagreements in the context of the evolution of stellar angular momentum. Finally, in Section 5 we draw our conclusions regarding the clusters' massive star populations. We plan to use the results of this work to examine the spectral energy distributions (SEDs) of the star + disk systems among the Be star population in a forthcoming paper.

\section{Observations}

We have obtained spectra for a total of 104 members of NGC 869 and NGC 884 during multiple observing runs: 2005 November using the Kitt Peak National Observatory (KPNO) Wisconsin Indiana Yale NOAO (WIYN) $3.5 \mathrm{~m}$ telescope with the Hydra multifiber spectrograph; 2010 August using the Wyoming Infrared Observatory (WIRO) $2.3 \mathrm{~m}$ telescope with the Long Slit spectrograph; 2011 November using the KPNO $2.1 \mathrm{~m}$ telescope with the GoldCam spectrograph; and 2012 January using the $0.9 \mathrm{~m}$ KPNO Coudé Feed (CF) telescope with the Coudé spectrograph. The UT dates, wavelength range, resolving power, number of targets, and instrumental setup details for all runs are summarized in Table 1.

All of the spectra obtained at the WIYN $3.5 \mathrm{~m}$ with the Hydra spectrograph have been zero corrected using standard routines in IRAF4, and have been flat-fielded, wavelength-calibrated, and skysubtracted in IRAF using the dohydra routine. The Hydra observations obtained by M. Virginia McSwain in 2005 consist of 7 exposures of NGC 869, totaling $2.25 \mathrm{hrs}$, and 5 exposures of NGC 884, totaling 2 hrs. Each exposure has been wavelength calibrated via a $\mathrm{CuAr}$ comparison spectrum both before and after the cluster observations. For each of the two configurations, the exposures have been

\footnotetext{
${ }^{4}$ IRAF is distributed by the National Optical Astronomy Observatory, which is operated by AURA, Inc., under cooperative agreement with the NSF.
}

transformed to a common heliocentric wavelength grid and co-added to produce good signal-to-noise for each star. The spectra were rectified to a unit continuum by fitting line-free regions.

Before any reduction or calibration routines were applied to the WIRO $2.3 \mathrm{~m}$ data, all spectra (object and comparison) were corrected for bit-flip errors with the rfits routine in IRAF. A CuAr calibration lamp source was used to obtain wavelength calibration spectra before and after every object spectrum. The spectra were then zero-corrected, flat-fielded, wavelength-calibrated, and rectified to a unit continuum using standard slit spectra routines in IRAF. Fainter objects that required multiple exposures were co-added prior to continuum rectification to improve signal-to-noise. Given the poorer resolution of blue WIRO spectra, we cannot use these data with our spectral fitting techniques described in Section 3. We therefore only use the blue and red WIRO spectra to identify Be stars exhibiting emission at the time of our observations.

Spectra from the KPNO $2.1 \mathrm{~m}$ instrument have been similarly zero-corrected, flat-fielded, and wavelength-calibrated using the standard routines found in IRAF. Once wavelength calibrated via the HeNeAr comparison lamp spectra, which were taken before and after every object spectrum, the data were rectified to a unit continuum.

In a similar manner, the spectra obtained with the $0.9 \mathrm{~m}$ KPNO CF telescope have been zerocorrected, flat-fielded, and wavelength-calibrated using the standard routines in IRAF. ThAr comparison spectra were taken every one to two hours during the run. The data were then rectified to a unit continuum.

\section{Physical Parameters from Spectral Mod- els}

The first component of our population study is to determine basic parameters for the cluster constituents using a methodology devised by McSwain et al. (2008). Using ground based optical spectroscopy (4000-5200 $\AA$ ) and model fitting techniques, we can determine $V \sin i, T_{\text {eff }}$, and $\log$ $g$ for each star. To obtain these measurements, we compare our observed spectra to grids of model Btype stars, determining a best fit to the data by minimizing the mean square of the deviations $\mathrm{rms}^{2}$.

We begin our analysis by making a rough esti- 
mate of $T_{\text {eff }}$ and $\log g$ for a star, and then we compare the He I $\lambda \lambda 4387,4471,4713$, and Mg II $\lambda 4481$ lines with the Kurucz ATLAS9 models Kurucz 1994) to determine $V \sin i$. These lines are used because their broadening is dominated by the effects of rotation, thus yielding a better indication of $V \sin i$. We then take a weighted average of the four values determined from each of the lines to give our measured value of $V \sin i$. The error, $\Delta V \sin i$, is determined by the offset from the measured best-fit value that increases the $\mathrm{rms}^{2}$ by $2.7 \mathrm{rms}^{2} / N$. Here, $N$ is the number of wavelength points within the fit region. A sample fit determined for He I $\lambda 4387$ in NGC 869-90 is shown in the bottom panel of Figure 1. Our results for $V \sin i$ and its errors are listed in columns 2 and 3 of Tables 2 and 3 ,

In Be stars it is possible that the He I lines may contain weak emission from the circumstellar disk, which may partially fill the absorption features and narrow the overall line profile. So while we have made measurements of $V \sin i$ where we can for Be stars in our sample, these values should be considered as lower limits.

We measure a mean $V$ sin $i=157 \mathrm{~km} \mathrm{~s}^{-1}$ with a standard deviation of $89 \mathrm{~km} \mathrm{~s}^{-1}$ for the normal B-type stars of both clusters, including binary systems. Assuming an average inclination angle of $i=60^{\circ}$, this gives a mean $V_{\mathrm{eq}}=181 \mathrm{~km} \mathrm{~s}^{-1}$ for our sample of B stars. For the Be stars we measure a mean $V \sin i=205 \pm 81 \mathrm{~km} \mathrm{~s}^{-1}$, with a mean $V_{\text {eq }}=237 \mathrm{~km} \mathrm{~s}^{-1}$. From this it is clear that the Be stars in these clusters are, on average, rotating somewhat more rapidly than their B-type counterparts.

The cumulative $V \sin i$ distributions for all Be and normal B-type stars in both NGC 869 and NGC 884 are shown in Figure 2, In comparison to other young open clusters (see McSwain et al. 2008, 2009), we find that the Be star population of NGC 869 and NGC 884 are rotating surprisingly more slowly that expected in comparison to their B-type counterparts. Using the two-sided KolmogorovSmirnov (K-S) statistical test, we investigate the null hypothesis that the distributions of B-type and Be stars differ. The K-S test indicates a $7.6 \%$ chance that the two populations are drawn from the same sample. Using the values of mass, $M_{\star}$, and radius, $R_{\star}$, discussed below for all stars in our sample, we can determine the critical velocity

$$
V_{\text {crit }}=\sqrt{\frac{G M_{\star}}{R_{\mathrm{e}}}}
$$

for our stars. For simplicity in this expression, we assume that the polar radius of the star, $R_{\mathrm{p}}$, is equal to $R_{\star}$, and that a rotationally distorted star has an equatorial radius $R_{\mathrm{e}}=1.5 R_{\mathrm{p}}$. With this, we find a mean $V_{\text {crit }}$ of $430 \mathrm{~km} \mathrm{~s}^{-1}$ for the B-type and Be stars in these clusters.

Having determined values of $V \sin i$ for each star, we turn again to model spectral fitting to determine values for $T_{\text {eff }}$ and $\log g$ from the $\mathrm{H} \gamma$ line at $4340 \AA$. The hydrogen Balmer lines are particularly sensitive to $T_{\text {eff }}$ and $\log g$, making them ideal for determining these quantities accurately. The method outlined here was employed for normal B-type stars and Be stars with no emission present in this work. For stars having $T_{\text {eff }} \leq 15000 \mathrm{~K}$ we employ the methods of Huang \& Gies (2006a), who use $\mathrm{H} \gamma$ line profiles generated by the line-blanketed, local thermodynamic equilibrium (LTE) Kurucz ATLAS9 and SYNSPEC codes. The "virtual star" models produced by their code simulate spherically symmetric stars with constant $T_{\text {eff }}$ and $\log g$ across their surface, and the spectra of these model stars are then used to measure these values and their errors of our observed spectrum, similar to our procedure for $V \sin i$. The errors $\Delta T_{\text {eff }}$ and $\Delta \log g$ are determined from the quadratic sum of the $V$ $\sin i$ propagated errors and the errors due to the intrinsic noise in the observed spectrum. For hotter stars, LTE models should systematically underestimate $T_{\text {eff }}$ as non-LTE effects alter the equivalent width of the $\mathrm{H} \gamma$ line we are measuring. Hence for stars having $T_{\text {eff }} \geq 15000 \mathrm{~K}$, we use instead the metal line-blanketed, non-LTE, plane-parallel, hydrostatic TLUSTY BSTAR2006 model spectra (Lanz \& Hubeny 2007). A sample fit of the $\mathrm{H} \gamma$ line in NGC 869-90 is shown in the top panel of Figure 1. The errors $\Delta T_{\text {eff }}$ and $\Delta \log g$ are determined from the values that produce an $\mathrm{rms}^{2}$ no more than $2.7 \mathrm{rms}^{2} / N$ greater than the minimum $\mathrm{rms}^{2}$. Our results for $T_{\text {eff }}, \log g$, and their respective errors are shown in columns $4-7$ of Table 2

$29 \%$ of the stars in our sample are rapid rotators, having measured values of $V \sin i$ in excess of $200 \mathrm{~km} \mathrm{~s}^{-1}$. At such significant rotational velocities, assuming a spherical shape for these stars is no longer plausible given the substantial centrifu- 
gal forces distorting the stars into oblate spheroids. This rotational distortion produces significant differences in both the temperatures and surface gravities at the polar and equatorial regions. As the measured values of $T_{\text {eff }}$ and $\log g$ are averages across the visible stellar hemisphere, these rotational effects produce lower values than expected, a phenomenon known as gravitational darkening. As the rotation rate for a star approaches its critical velocity, the equatorial radius may increase by as much as $50 \%$, while the polar radius remains unchanged. For these reasons, we convert our measured $\log g$ to $\log g_{\text {polar }}$, as detailed in Huang \& Gies (2006a). The authors produce detailed spectroscopic models to investigate the effects of such rotational distortions and determine a statistical correction factor for $\log g$. This factor is averaged over all possible values of inclination angle, $i$, for a variety of stellar models. By bilinearly interpolating between their models, we converted our measured value of $\log g$ to $\log g_{\text {polar }}$. This value of $\log g_{\text {polar }}$ is a better measure of the true surface gravity of the star, untainted by the effects of rapid rotation, and provides us a more accurate means of comparison between slowly and rapidly rotating stars. The $\log g_{\text {polar }}$ correction is therefore most significant for stars rotating more rapidly than $50 \%$ of $V_{\text {crit }}$. We assume that this conversion produces a negligible change to the value determined previously for $\Delta \log g$. Our derived values of $\log g_{\text {polar }}$ are listed in column 8 of Table 2

Once we have measured parameters for all of the B-type stars, we can then determine $M_{\star}$ and $R_{\star}$ for each of them by interpolating values from the Schaller et al. (1992) non-rotating evolutionary tracks, consistent with the slow rotation of most of our targets. These evolutionary tracks are shown plotted with $T_{\text {eff }}$ and $\log g_{\text {polar }}$ in Figure 5. The errors $\Delta M_{\star}$ and $\Delta R_{\star}$ correspond to our measured $\Delta T_{\text {eff }}$ and $\Delta \log g$. Additionally, we have compared our results with the rotating models of Ekström et al. (2012), and we find agreement between the models to within $10 \%$. The resulting values of $M_{\star}, R_{\star}$, and their respective errors are listed in columns 9-12 of Tables 2 and 3. We have also checked the accuracy of our results by comparing the TLUSTY BSTAR2006 model SEDs with the observed SEDs for our B-type sample stars, and find excellent agreement between our derivations of distance and reddening with the accepted values of Currie et al. (2010). These results will be discussed further in a forthcoming paper.

We note that star NGC 869-566 did not show any signs of emission in our initial observations, but has since exhibited progressively stronger emission in our more recent 2010 and 2012 observations; hence we include it among the non-emission Be stars and have measured $T_{\text {eff }}$ and $\log g$ from the $\mathrm{H} \gamma$ line it its 2005 blue spectrum. For the other Be stars in our sample we cannot determine accurate values for $T_{\text {eff }}$ and $\log g$ via the same model fitting technique, as hydrogen absorption line profiles are altered by emission during disk phases of these stars. Instead we can use Strömgren photometry available from the WEBDA 5 database to correlate $T_{\text {eff }}$ and $\log g$ for all of the B-type and Be stars in our sample. Nonemission B stars with both available Strömgren photometry and spectral model fits were included as calibration stars for the photometric technique. To this we add eight additional stars from Napiwotzki et al. (1993) with well-known $T_{\text {eff }}$ and available Strömgren data. We use ubvy magnitudes to first determine the Strömgren indices $m_{1}$ and $c_{1}$, given by

$$
\begin{aligned}
m_{1} & =(v-b)-(b-y) \\
c_{1} & =(u-v)-(v-b) .
\end{aligned}
$$

The calculation of $T_{\text {eff }}$ determined by Balona (1984) requires us to convert the $c_{1}$ index to the dereddened index $c$ via the expressions

$$
\begin{aligned}
E(b-y) & =E(B-V) \times 0.754 \\
c_{0} & =c_{1}-0.19[E(b-y)] \\
c & =\log \left(c_{0}+0.200\right),
\end{aligned}
$$

which use the reddening values to the clusters, $\mathrm{E}(B-V)=0.55$ and $\mathrm{E}(B-V)=0.52$ for $h$ and $\chi$ Per, respectively (Currie et al. 2010; Bragg \& Kenyon 2005). Using these indices and the $\mathrm{H} \beta$ line magnitude $(\beta)$, we can then calculate $T_{\text {eff }}$ via the relationship

$$
\begin{array}{r}
\log T_{\text {Balona }}=3.9036-0.4816(c)-0.5290(\beta) \\
-0.1260(c)^{2}+0.0924(\beta)(c)-0.4013(\beta)^{2}
\end{array}
$$

given by Balona (1984).

McSwain et al. (2008) found that this calculated value, $T_{\text {Balona }}$, slightly underestimates the true $T_{\text {eff }}$ of the B-type stars. Thus, we performed a linear

${ }^{5}$ Available online at www.univie.ac.at/webda and maintained by Ernst Paunzen 
fit to the data, shown in Figure 3, and determined a correction factor that will bring the two independent measurements into agreement. In this way, we are able to use the B-type stars measured by both methods as a calibration to yield values of $T_{\text {eff }}$ for the otherwise immeasurable Be stars. These $T_{\text {eff }}$ and our calculated errors are listed in columns 4 and 5 of Table 3 .

To determine $\log g$, Balona (1984) advocate using $\beta$ and $c_{0}$. Given that $\beta$ serves as an indicator of $\log g$ via spectra line width, and $c_{0}$ is an indicator of temperature in hot stars, the relationship between these two values can serve as a HertzsprungRussell diagram for the cluster providing a means for investigating the temperature and evolutionary trends of the stellar population. In more evolved giant or supergiant stars (luminosity classes III and I, respectively), the hydrogen lines are very narrow due to the lower densities in the outer atmospheres of these stars, decreasing the collisional rates that produce the pressure broadening mechanism. Main sequence stars (luminosity class $\mathrm{V}$ ), which host more dense atmospheres and thus higher collisional rates and higher pressures, have broader hydrogen lines. In general, we do see that the values of $c_{0}$ and $\beta$ for the B-type calibrators shown in Figure 4 agree with the relations for class V and III stars of Balona \& Shobbrook (1984). However, the circumstellar disks present in Be stars (shown as filled diamonds in Figure 4) will artificially brighten the $\beta$ magnitudes of these stars, contaminating the $c_{0}-\beta$ relation for these stars and our calculated values of $\log g$. The spread in $\log g$ of the B-type calibration stars prevents us from simply applying either of the $c_{0}-\beta$ relations shown in Figure 4 to the population. Instead, we perform a linear fit to the calibration star data and obtain a corrected value of $\beta$ which we then use to correct our calculated $T_{\text {Balona }}$ and finalize our calibrated, calculated temperatures and surface gravities for the Be stars exhibiting emission in our data. Since the clusters are approximately the same age, this single fit is appropriate. Additional details regarding the method to determine $T_{\text {eff }}, \log$ $g, M_{\star}, R_{\star}$, and their respective errors for the $\mathrm{Be}$ stars can be found in McSwain et al. (2008). We do not perform any further correction to obtain log $g_{\text {polar }}$ for the Be stars measured with this technique, given the large scatter between the calibrators' log $g_{\text {polar }}$ and their Strömgren $\log g$. The final results for $\log g$ and $\Delta \log g$ of the Be stars are listed in columns $6-7$ of Table 3

We highlight our results regarding the Be star population in Table 3, and we note that these Be stars were selected from the literature based on previous observations of spectral line emission or via photometric surveys needing spectroscopic confirmation. Presented within Table 3 are a total of 28 known or proposed Be stars within our sample of 104 stars. For these objects we were able to examine the state of emission from our observations, and then determine their stellar parameters by our spectral modeling or Strömgren photometry methods as detailed earlier in this section. The broad-band Strömgren indices used to derive their $T_{\text {eff }}$ and $\log g$ are not likely to be affected by their rapid rotation. We do see evidence of emission in 22 of these Be stars. Interestingly, we see evidence in our data for at least 8 "transient" Be stars (McSwain et al. 2008). The stars NGC 869-146, NGC 869-717, NGC 8691268, and NGC 884-2262 were observed as Be stars in the past (Slettebak 1985; Fabregat et al. 1994; Keller et al. 2001; Bragg \& Kenyon 2002), and the star NGC $884-2468$ is a proposed candidate Be star (Currie et al. 2008). However, we do not see evidence of emission in our observations of these objects. As mentioned previously, the known Be star NGC 869-566 was initially observed by us in 2005 and showed no sign of emission in its spectrum. However, it has since developed increasingly stronger emission in our 2010 and 2012 observations. For the other two transient Be stars (NGC 869-49, NGC 884-1772), we do see emission in our spectra, however other authors have noted them in non-emission phases in the past (Schild 1966; Slettebak 1985; Bragg \& Kenvon 2002; Keller et al. 2001). We are currently preparing a followup paper that discusses the $\mathrm{H} \alpha$ emission properties of all the Be stars in our sample.

\section{Comparison with Other Studies}

Previous studies, such as Strom et al. (2005) and Huang \& Gies (2006a), have investigated some of our B star targets to determine the same basic stellar parameters we do, however their analyses employed LTE atmospheric models or quantitative corrections to LTE model measurements to account for non-LTE effects. Between these two studies there are clear discrepancies in their measurements and 
the conclusions each draws regarding the natal rotation rates and angular momentum evolution of the clusters' massive stars. An accurate determination of a given star's surface gravity is essential to the evaluation of stellar radius and evolutionary state. As we show here, non-LTE effects can contribute to significant errors in measurements of $\log g$.

Amongst the 54 stars common to our sample and that of Huang \& Gies (2006a), we find some discrepancy between our respective results. As can be seen in Figure 6, there is very good agreement in our determinations of $V \sin i$. This is to be expected since we both used LTE models to fit the He I and Mg II lines to measure $V \sin i$. The differences at low $V \sin i$ may be due to a difference in spectral resolution between our respective datasets.

However, differences in our measured temperatures for stars with $T_{\text {eff }} \geq 15000 \mathrm{~K}$ and in our measured $\log g$ values are clearly apparent in Figures 7 and 8, respectively. Given that our general methodology for measuring $T_{\text {eff }}$ and $\log g$ is the same as Huang \& Gies (2006a), the source of these discrepancies lies partially in our use of different stellar models. While Huang \& Gies (2006a) employ the LTE Kurucz ATLAS9 models (Kurucz 1994), we use the more recently available non-LTE TLUSTY BSTAR2006 models of Lanz \& Hubeny (2007) for those stars in our sample with $T_{\text {eff }} \geq 15000 \mathrm{~K}$. In their analysis Huang \& Gies (2006a) acknowledge that their derived temperatures are likely to be slightly lower and gravities slightly higher compared to measurements derived from non-LTE model atmospheres, as shown by the comparative analysis of Lanz \& Hubenv (2007).

Lanz \& Hubeny (2007) find that in the nonLTE models the hydrogen Balmer lines tend to be broader and stronger due to the overpopulation of the $n=2$ energy state, thus LTE models will yield overestimated surface gravities due to the altered the shape of the Balmer line wings. Przybilla et al. (2011) compared LTE ATLAS9 models to non-LTE TLUSTY models for temperatures between 15000$35000 \mathrm{~K}$. They found that non-LTE effects are significant above 22,000 K, affecting both the cores and wings of the Balmer lines. They find that LTE Balmer line profiles have equivalent widths up to $30 \%$ lower than in non-LTE line profiles. This would cause a non-LTE model to find a higher temperature for the same observed line, or LTE models underestimate the temperature. Przybilla et al. (2011) also find that LTE models of the $\mathrm{H} \gamma$ line may overestimate $\log \mathrm{g}$ by up to 0.2 dex.

While the expected temperature disagreement is opposite of the trend we find in our comparison of our work with Huang \& Gies (2006a) results, several of our common sample stars with temperatures greater than $24,000 \mathrm{~K}$ are Be stars, some of which we find to exhibit transient behavior. It is likely that emission has subtly filled in or otherwise altered the $\mathrm{H} \gamma$ line profile, which would result in the overestimation of $T_{\text {eff }}$ for these stars by Huang \& Gies (2006a). We also find one proposed spectroscopic binary among this common sample, so the $\mathrm{H} \gamma$ line profiles may be further altered by variable line blending effects. For the remaining B-type stars in this region it is possible that our temperature discrepancy is due to variable emission in unknown Be stars, unresolved binaries, clumping in the hot stellar wind, or differences in the atomic species included in our respective atmospheric models that affect hydrogen stark broadening (Przybilla \& Butler 2004).

With the quantified disagreement in $T_{\text {eff }}$ and $\log$ $g$ shown here, the anticipated effect of non-LTE atmospheres on the measurement results is more significant than initially assumed by Huang \& Gies (2006a). The effects of these overestimated temperatures and gravities also affect their determined stellar masses, their usage of $\log g$ as an indicator of stellar evolutionary status, and their determined spin-down rates of $\mathrm{B}$ and Be stars. We find that cluster members are more evolved than indicated by Huang \& Gies (2006a). These B stars may spin down more slowly than the rates observed by Huang \& Gies (2006a) and Huang et al. (2010).

We also compare our results to those presented by Strom et al. (2005), who adopt the $T_{\text {eff }}$ values derived from UBV photometry by Slesnick et al. (2002). In their study they find reasonable agreement between their measurements of $V \sin i$ and those of Huang \& Gies (2006a), though their results are systematically $5 \%$ smaller than the results of Huang \& Gies (2006a). Comparing the results of the 26 stars common to our two samples, we find a similar agreement and systematic underestimation of Strom et al. 's $V \sin i$ values when compared to our measurements, as is expected given the excellent agreement of our results with those of Huang \& Gies (2006a). Upon further comparison of our results, we find that Strom et al. (2005) and Slesnick et al. (2002) have overestimated $T_{\text {eff }}$ for hotter stars as 
well. We note that the two most discrepant stars are both Be stars, suggesting that their $\mathrm{H} \beta$ emission contaminates the B-band brightness used to derive $T_{\text {eff }}$. Thus $M_{\star}$ and $R_{\star}$ for the Be stars as shown in Figure 9 are also likely overestimated.

Finally, we note that many of our Be stars were found to be possible spectroscopic binaries by Huang \& Gies (2006a) and Strom et al. (2005). Since Huang \& Gies (2006a) did not present measurements of $T_{\text {eff }}$ or $\log g$ for many of their spectroscopic binaries, not all of our measurements could be directly compared. Their classification as binaries may be inaccurate due to variable emission in their spectral lines. Further monitoring of their radial velocities as well as their emission will clarify their status.

\section{Conclusions and Future Work}

We have measured $V \sin i, T_{\text {eff }}, \log g_{\text {polar }}, M_{\star}$, and $R_{\star}$ for $104 \mathrm{~B}$-type and Be star members of NGC 869 and NGC 884 using spectroscopic modeling techniques and calculations from Strömgren photometry. Our determined values for $V \sin i$ are in good agreement with the earlier results of Huang \& Gies (2006a), though there is some discrepancy in our measured temperatures and surface gravities due to our use of the more recently available non-LTE BSTAR2006 stellar models of Lanz \& Hubeny (2007) and the possible contamination of Be stars and spectroscopic binaries. Because of the resulting over-estimation of $\log g$, Huang \& Gies (2006a) have underestimated the retention of initial angular momentum by the cluster members.

We find that the cluster members are significantly more evolved than found by previous measurements. We also identify 8 transient Be stars in $h$ and $\chi$ Per. The Be stars in these clusters are also rotating more slowly than expected based upon other young open clusters. Further monitoring of the massive stellar constituents of these clusters, and their rotation rates is well warranted.

In a forthcoming paper we will examine our sample of Be stars and their disk structures in greater detail. We will use the SEDs of B-type cluster members to compare our derived distances with previous measurements. Using our determined stellar parameters, we will be able to separate and examine the stellar and disk contributions to the total Be star
+ disk system flux through their observed SEDs. From these SEDs and multiple observations of $\mathrm{H} \alpha$ we will investigate the Be disk radii, masses, and longterm variability.

We are grateful to the anonymous referee for comments that greatly improved this manuscript. We would like to thank Yale University for providing access to the WIYN telescope at KPNO. We are grateful to the University of Wyoming for providing observing time at the WIRO $2.3 \mathrm{~m}$ telescope, as well as to Chip Kobulnicky and Dan Kiminki for their support of our observations. We are very grateful to the support staff at NOAO, especially Di Harmer and Daryl Wilmarth for their help in obtaining our observations at KPNO. We are also very appreciative of the helpful feedback regarding this work provided by Wenjin Huang and Doug Gies. This research has made use of the WEBDA database, operated at the Institute for Astronomy of the University of Vienna. This work has been supported by the National Science Foundation under grant AST-1109247 and by NASA under DPR numbers NNX08AX79G, NNX09AP86G, and NNX11AO41G. M.V.M. was supported by an NSF Astronomy and Astrophysics Postdoctoral Fellowship under award AST-0401460. C.A. and A.N.M.B. are grateful for thesis student travel support provided by NOAO. A.N.M.B. is also supported by a Grant-In-Aid of Research (G20110315157195) from the National Academy of Sciences, administered by Sigma Xi. We are very grateful for National Science Foundation support provided for B.O. through REU site grant PHY0353620 to Lehigh University. Institutional support was provided by Lehigh University.

Facilities: WIRO (), KPNO:CFT (), KPNO:2.1m (), WIYN ()

\section{REFERENCES}

Adams, W. S., \& VanMaanen, A. 1913, AJ, 27, 187

Balona, L. A. 1984, MNRAS, 211, 973

Balona, L. A., \& Shobbrook, R. R. 1984, MNRAS, 211, 375

Bragg, A., \& Kenyon, S. 2002, AJ, 124, 3289

—. 2005, AJ, 130, 134 
Cranmer, S. R. 2009, ApJ, 701, 396

Currie, T., et al. 2008, ApJ, 672, 558

—. 2010, ApJS, 186, 191

Ekström, S., et al. 2012, A\&A, 537, 146

Emilio, M., et al. 2010, A\&A, 522, 43

Fabregat, J., Torrejón, J. M., \& Bernabeu, G. 1994, Be Star Newsl, 29, 8

Hertzsprung, E. 1922, BAN, 1, 151

Huang, W., \& Gies, D. R. 2006a, ApJ, 648, 591

—. 2006b, ApJ, 648, 580

Huang, W., Gies, D. R., \& McSwain, M. V. 2010, ApJ, 722, 605

Keller, S. C., Grebel, E. K., Miller, G. J., \& Yoss, K. M. 2001, AJ, 122, 248

Krzesiński, J., \& Pigulski, A. 1997, A\&A, 325, 987

Krzesiński, J., Pigulski, A., \& Kołaczkowski, Z. 1999, A\&A, 345, 505

Kurucz, R. 1994, Solar abundance model atmospheres for $0,1,2,4,8 \mathrm{~km} / \mathrm{s}$. Kurucz CD-ROM No. 19. Cambridge, Mass.: Smithsonian Astrophysical Observatory, 1994., 19

Lanz, T., \& Hubeny, I. 2007, ApJS, 169, 83

McSwain, M. V., Huang, W., \& Gies, D. R. 2009, ApJ, 700, 1216

McSwain, M. V., Huang, W., Gies, D. R., Grundstrom, E. D., \& Townsend, R. H. D. 2008, ApJ, 672,590

Messow, B. 1913, AAHam, 2b, 1M

Meynet, G., \& Maeder, A. 2000, A\&A, 361, 101

Napiwotzki, R., Schoenberner, D., \& Wenske, V. 1993, A\&A, 268, 653

Porter, J. M., \& Rivinius, T. 2003, PASP, 115, 1153

Przybilla, N., \& Butler, K. 2004, ApJ, 609, 1181

Przybilla, N., Nieva, M., \& Butler, K. 2011, JPhCS, 328, 012015
Rivinius, T., Baade, D., \& S̆tefl, S. 2003, A\&A, 411, 229

Rivinius, T., et al. 2001, A\&A, 369, 1058

Saesen, S., et al. 2010, A\&A, 515, A16

Schaller, G., Schraerer, D., Meynet, G., \& Maeder, A. 1992, A\&AS, 96, 269

Schild, R. E. 1966, ApJ, 146, 142

Slesnick, C., Hillenbrand, L. A., \& Massey, P. 2002, ApJ, 576, 880

Slettebak, A. 1968, ApJ, 154, 933

—. 1985, ApJ, 59, 769

Southworth, J., Zucker, S., Maxted, P. F. L., \& Smalley, B. 2004, MNRAS, 355, 986

Strom, S. E., Wolff, S. C., \& Dror, D. H. A. 2005, AJ, 129, 809

Trumpler, R. J. 1926, PASP, 38, 350

This 2-column preprint was prepared with the AAS IATEX macros v5.2. 

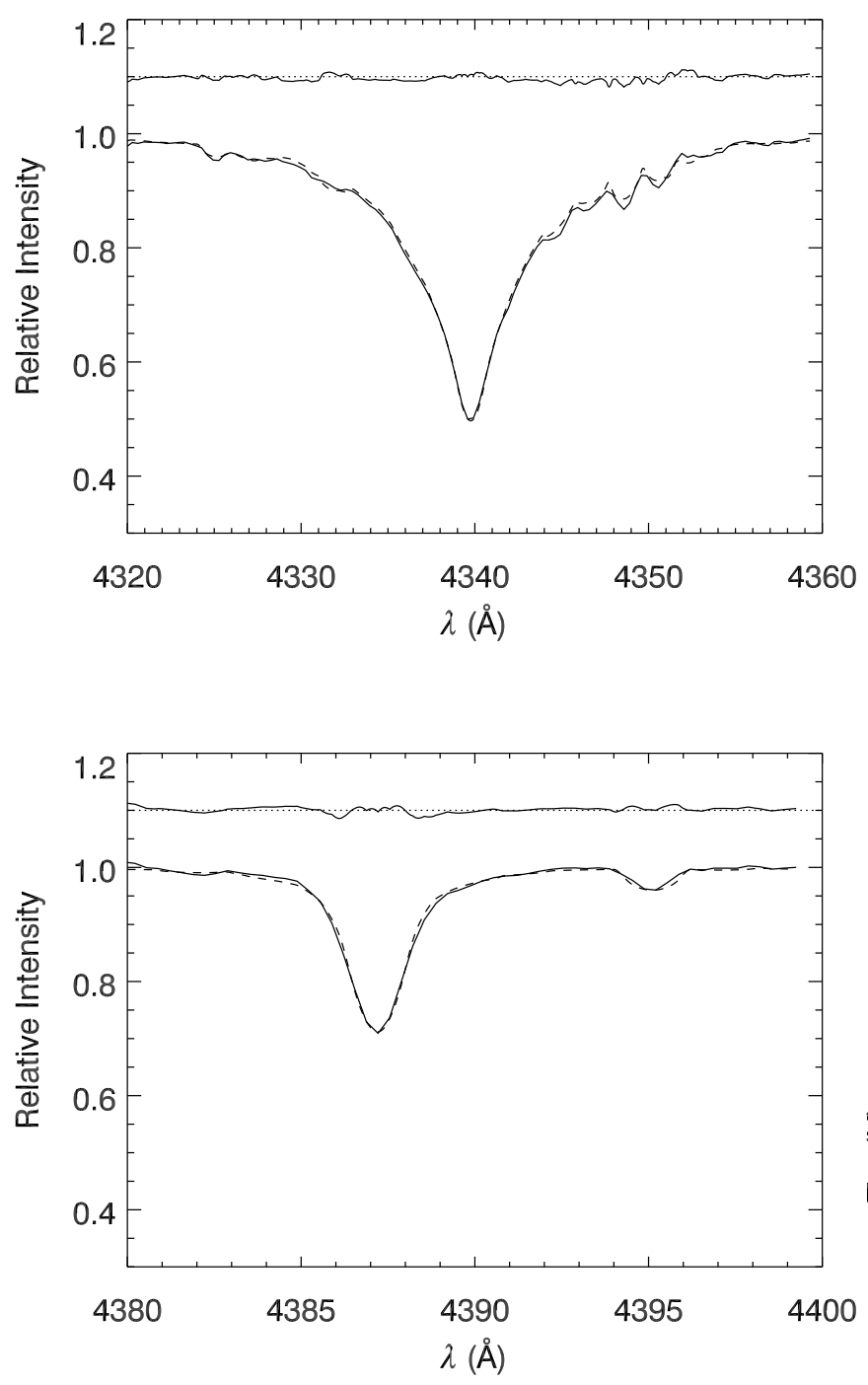

Fig. 1. - Sample spectral line fits for NGC 869-90. Shown on the top is $\mathrm{H} \gamma$ and on the bottom is He I $\lambda 4378$. The solid line is our observed spectrum while the dashed line displays our model fit to the line, with the computed residual shown above, shifted for clarity.

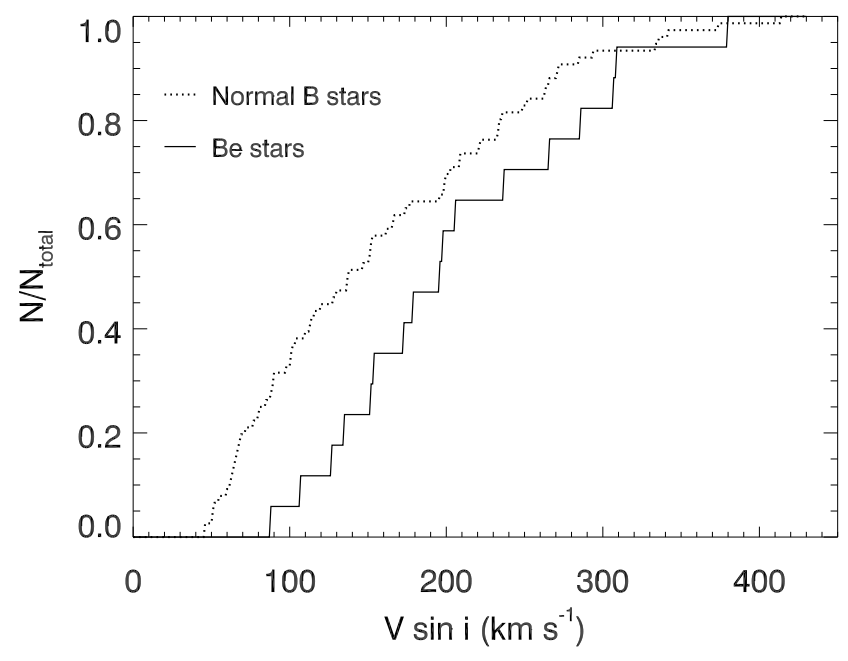

Fig. 2.- Cumulative distribution function of $V \sin$ $i$ for the Be stars (dashed line) and the normal Btype stars (dotted line) of both NGC 869 and NGC 884.

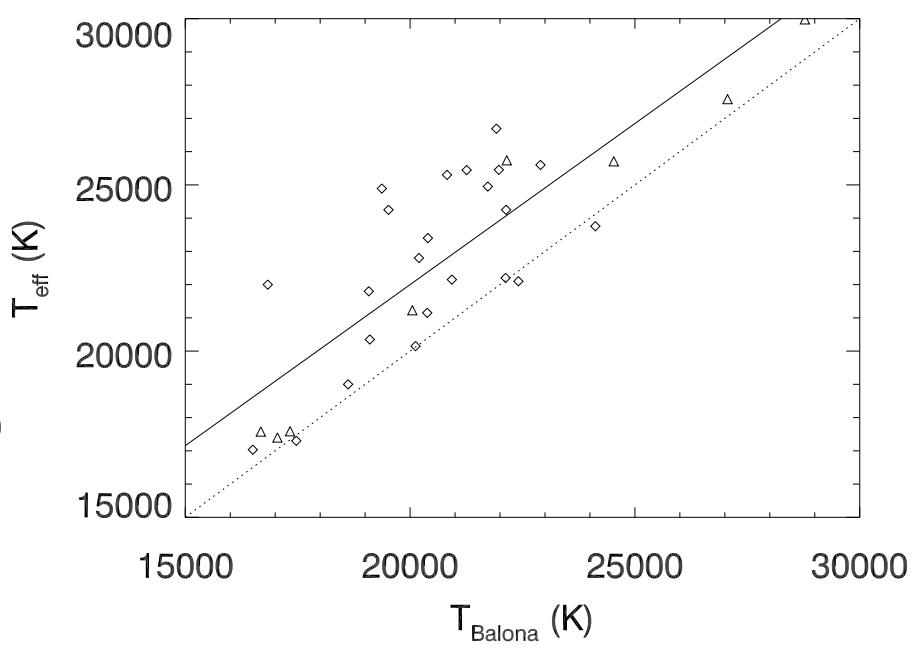

Fig. 3.- $T_{\text {eff }}$ measured for the B-type temperature calibration stars from our work (diamonds) and from Napiwotzki et al. (1993)(triangles) compared to their calculated $T_{\text {Balona }}$ (Balona 1984). A linear fit of the two temperature scales (solid line) and the 1:1 agreement (dotted line) are also shown. 


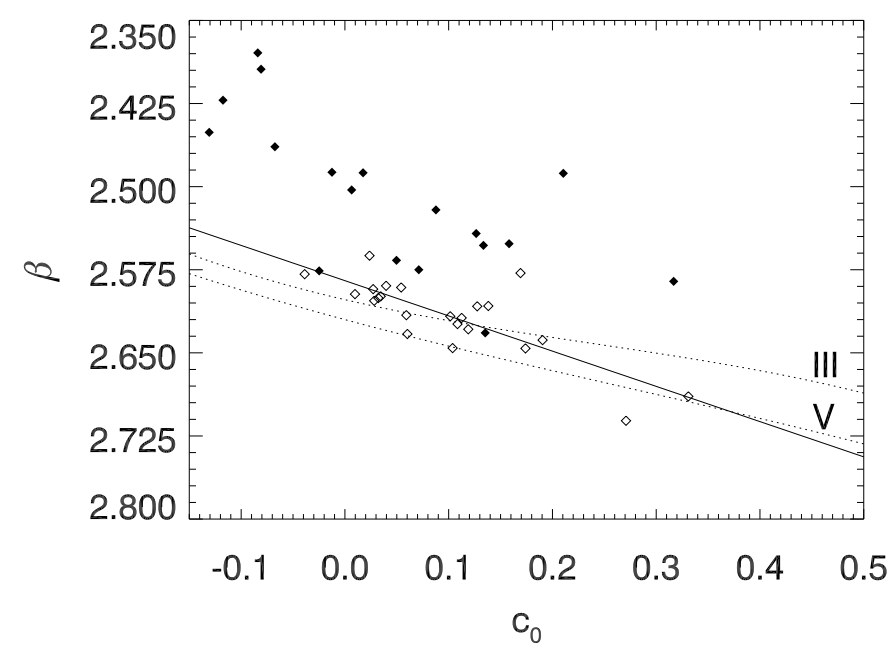

Fig. 4.- Strömgren $c_{0}$ index and $\beta$ magnitude are plotted with the $c_{0}-\beta$ relations for luminosity class V and III stars (Balona \& Shobbrook 1984; dotted lines). The B-type temperature calibration stars from this work (diamonds) are plotted with fifteen Be stars (filled diamonds) to demonstrate that the Be star $\beta$ magnitudes are brightened due to the disk emission present. A best fit line for the calibration stars is also shown (solid line).
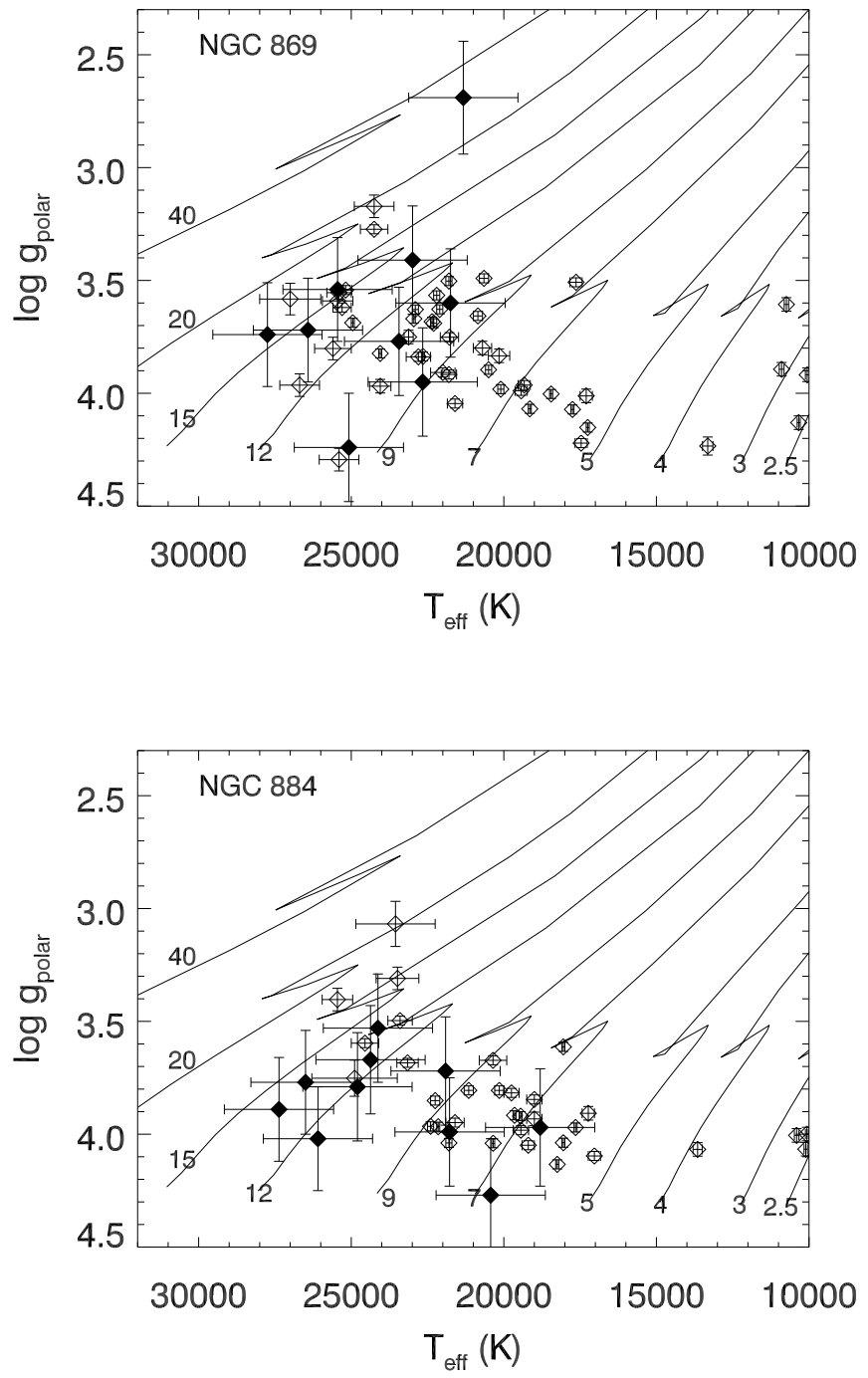

Fig. 5.- For both NGC 869 (top) and NGC 884 (bottom), $T_{\text {eff }}$ and $\log g_{\text {polar }}$ are plotted with the evolutionary tracks of Schaller et al. (1992). The zero age main sequence (ZAMS) mass of each evolutionary track is labeled along the bottom. Normal B-type stars are shown as open diamonds while Be stars are filled diamonds. 


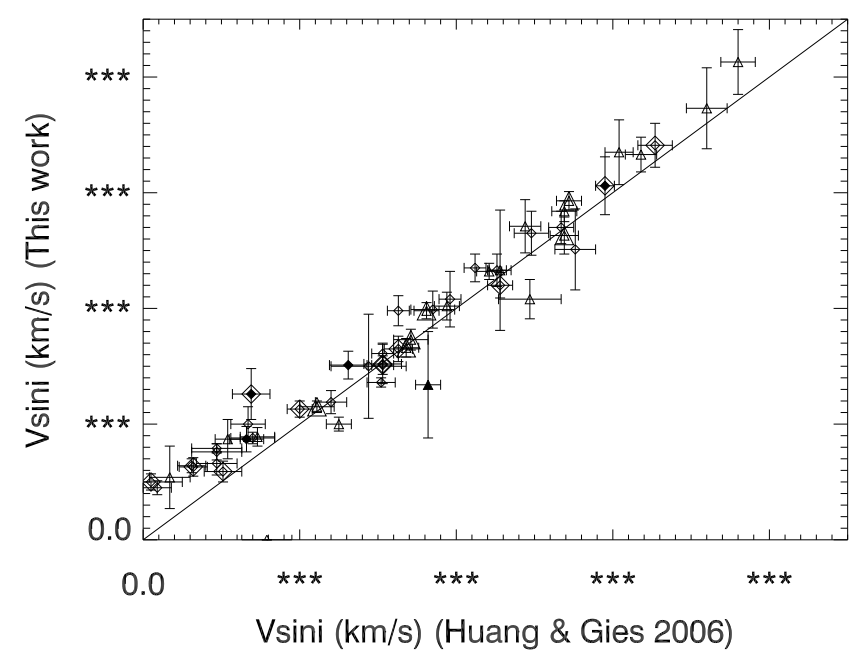

Fig. 6. - Comparison of our $V \sin i$ measurements with those of Huang \& Gies (2006a). NGC 869 cluster members are shown as open diamonds, while NGC 884 members are shown as open triangles. Be stars are shown as filled diamonds and triangles. Spectroscopic binaries, as noted in Tables 2 and 3, are highlighted by double-sized symbols.

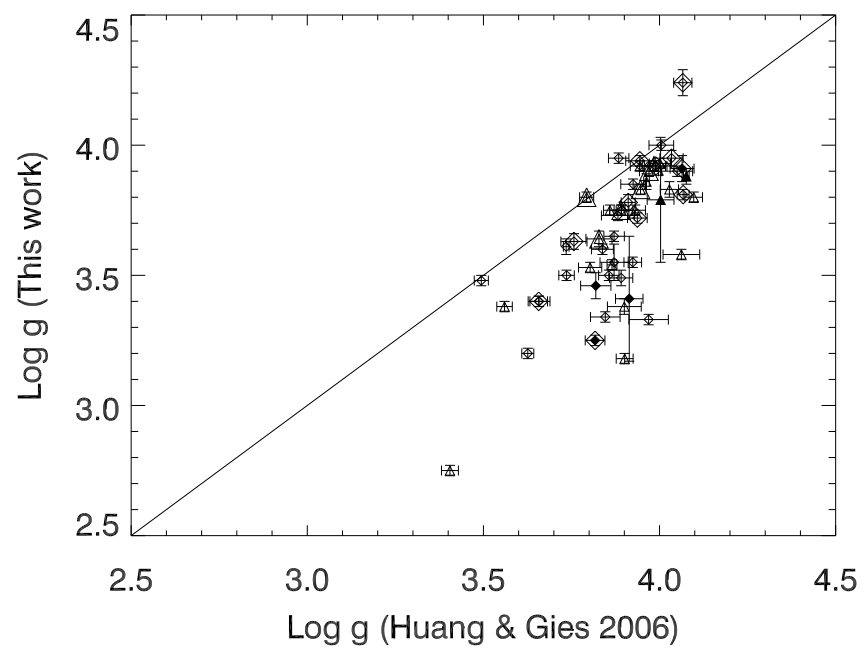

Fig. 8.- Comparison of our $\log g$ measurements with those of Huang \& Gies (2006a), in same format as Figure 6.

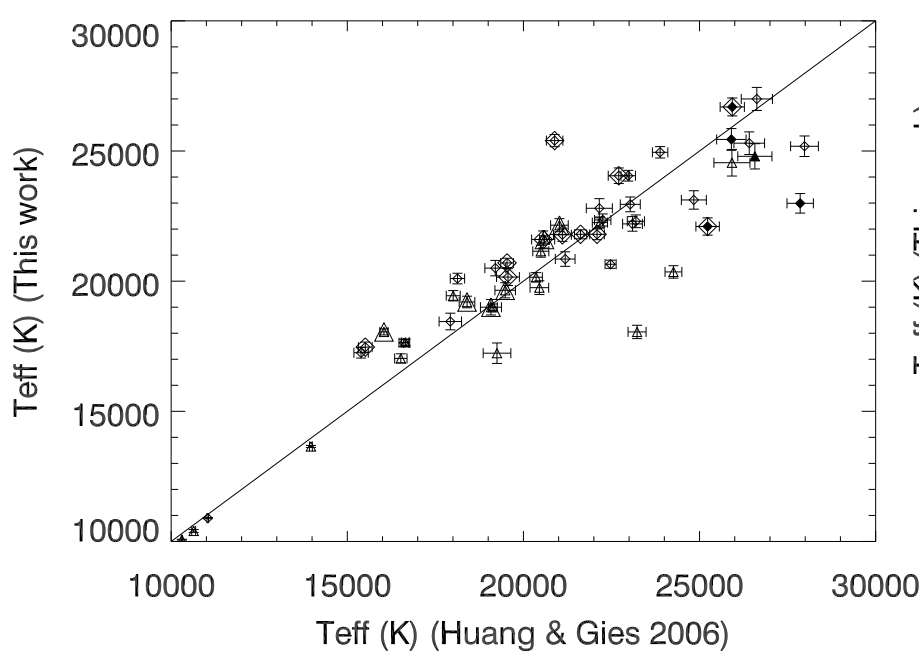

Fig. 7.- Comparison of our $T_{\text {eff }}$ measurements with those of Huang \& Gies (2006a), in same format as Figure 6 .

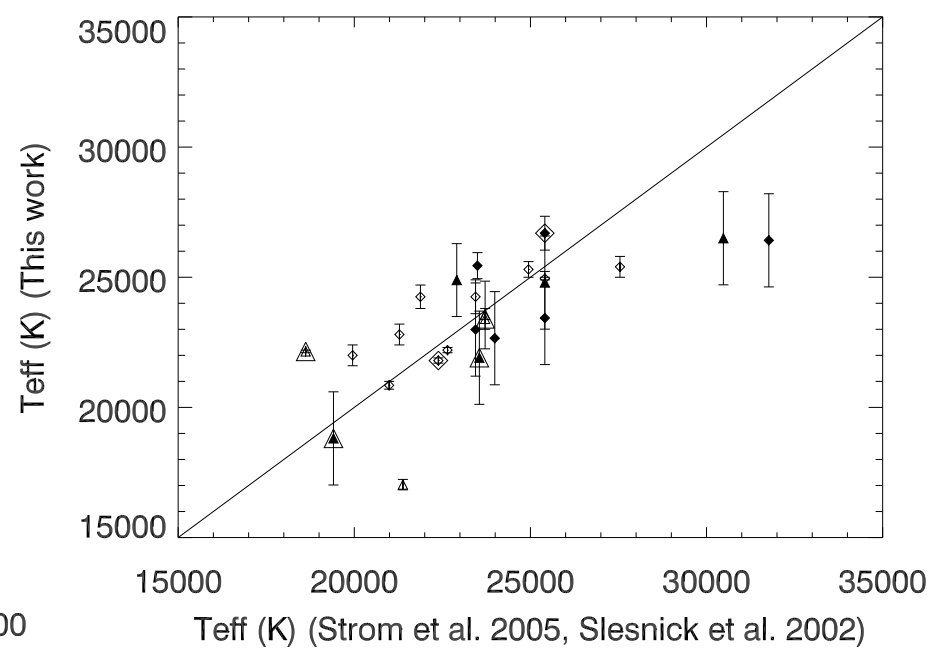

Fig. 9.- Comparison of our $T_{\text {eff }}$ measurements with those of Strom et al. (2005) and Slesnick et al. (2002), in same format as Figure 6. 
Table 1: Journal of Spectroscopy

\begin{tabular}{|c|c|c|c|c|c|c|c|}
\hline UT Dates & $\begin{array}{c}\text { Range } \\
(\AA)\end{array}$ & $\begin{array}{c}\text { Resolving Power } \\
(\lambda / \Delta \lambda)\end{array}$ & $\begin{array}{l}\text { Number of } \\
\text { Targets }\end{array}$ & $\begin{array}{l}\text { Telescope/ } \\
\text { Spectrograph }\end{array}$ & $\begin{array}{c}\text { Grating/ } \\
\text { Order }\end{array}$ & Filter & $\begin{array}{l}\text { Camera/ } \\
\text { Detector }\end{array}$ \\
\hline 2005 Nov $14-15$ & $4250-4900$ & 4700 & 92 & WIYN $3.5 \mathrm{~m} /$ Hydra & $1200 @ 28.7 / 2$ & BG39 & Red bench, blue fibers / 5TA1 \\
\hline 2010 Aug $23-27$ & $4000-5200$ & 1600 & 2 & WIRO $2.3 \mathrm{~m}$ / Long Slit & $\mathrm{LS}-1 / 2$ & BG40 & — / SBIG-ST-2000 \\
\hline 2010 Aug $28-31$ & $5350-6810$ & 4500 & 6 & WIRO $2.3 \mathrm{~m}$ / Long Slit & $\mathrm{LS}-2 / 1$ & GG455 & - / SBIG-ST-2000 \\
\hline 2011 Nov $10-13$ & $4000-5000$ & 2300 & 1 & KPNO $2.1 \mathrm{~m} /$ GoldCam & $47 / 2$ & CuSO4, WG354 & Gold / F3KC \\
\hline 2012 Jan $3-8$ & $4090-4550$ & 7800 & 2 & KPNO CF/Coudé & $\mathrm{B} / 3$ & 4-96 & Cam5 / T2KB \\
\hline 2012 Jan 3-8 & $6320-7025$ & 11700 & 1 & KPNO CF/Coudé & $\mathrm{B} / 2$ & OG-55 & Cam5 / T2KB \\
\hline
\end{tabular}


TABLE 2

Physical Parameters of B-type Cluster Members

\begin{tabular}{|c|c|c|c|c|c|c|c|c|c|c|c|c|}
\hline & $\begin{array}{c}\text { WEBDA } \\
\text { ID } \\
(1) \\
\end{array}$ & $\begin{array}{c}V \sin i \\
\left(\mathbf{k m ~ \mathbf { s } ^ { - 1 }}\right) \\
(2)\end{array}$ & $\begin{array}{c}\Delta V \sin i \\
\left(\mathbf{k m ~ \mathbf { s } ^ { - 1 }}\right) \\
(3)\end{array}$ & $\begin{array}{l}T_{\text {eff }} \\
(\mathbf{K}) \\
(4) \\
\end{array}$ & $\begin{array}{c}\Delta T_{\text {eff }} \\
(\mathbf{K}) \\
(5) \\
\end{array}$ & $\begin{array}{c}\log g \\
\left(\begin{array}{c}\text { dex }) \\
(6)\end{array}\right. \\
\end{array}$ & $\begin{array}{c}\Delta \log g \\
(\text { dex }) \\
(7)\end{array}$ & $\begin{array}{c}\log g_{\text {polar }} \\
(\text { dex }) \\
(8)\end{array}$ & $\begin{array}{c}M_{\star} \\
\left(M_{\odot}\right) \\
(9) \\
\end{array}$ & $\begin{array}{c}\Delta M_{\star} \\
\left(M_{\odot}\right) \\
(10) \\
\end{array}$ & $\begin{array}{c}R_{\star} \\
\left(R_{\odot}\right) \\
(11) \\
\end{array}$ & $\begin{array}{c}\Delta R_{\star} \\
\left(R_{\odot}\right) \\
(12) \\
\end{array}$ \\
\hline & NGC 869 & & & & & & & & & & & \\
\hline & $63^{\mathrm{a}}$ & 151 & 8 & 21800 & 250 & 3.81 & 0.02 & 3.92 & 8.7 & 0.1 & 5.4 & 1.7 \\
\hline & 87 & 89 & 16 & 10060 & 60 & 3.83 & 0.03 & 3.92 & 2.8 & 0.1 & 3.0 & 0.3 \\
\hline & 90 & 64 & 6 & 22350 & 50 & 3.65 & 0.02 & 3.68 & 10.7 & 0.1 & 7.8 & 1.4 \\
\hline & $133^{\mathrm{a}}$ & 341 & 19 & 17500 & 200 & 3.92 & 0.02 & 4.22 & 5.4 & 0.1 & 3.0 & 1.2 \\
\hline & 138 & 97 & 12 & 19400 & 200 & 3.93 & 0.02 & 3.99 & 6.9 & 0.1 & 4.4 & 1.3 \\
\hline & 197 & 262 & 25 & 10340 & 51 & 3.89 & 0.03 & 4.13 & 2.6 & 0.1 & 2.3 & 0.1 \\
\hline & 250 & 109 & 25 & 17750 & 50 & 4.00 & 0.02 & 4.07 & 5.9 & 0.1 & 3.7 & 1.1 \\
\hline & 260 & 198 & 13 & 25200 & 200 & 3.33 & 0.02 & 3.54 & 14.9 & 0.3 & 10.8 & 2.0 \\
\hline & 289 & 66 & 10 & 20100 & 100 & 3.95 & 0.02 & 3.98 & 7.4 & 0.1 & 4.6 & 1.4 \\
\hline & $323^{\mathrm{a}}$ & 50 & 7 & 20700 & 300 & 3.78 & 0.03 & 3.80 & 8.6 & 0.2 & 6.1 & 1.2 \\
\hline & 339 & 76 & 7 & 27000 & 1000 & 3.55 & 0.07 & 3.58 & 17.2 & 1.5 & 11.1 & 3.0 \\
\hline & 350 & 136 & 4 & 18450 & 50 & 3.90 & 0.02 & 4.00 & 6.4 & 0.1 & 4.2 & 1.1 \\
\hline & 380 & 112 & 6 & 19150 & 50 & 4.00 & 0.02 & 4.07 & 6.6 & 0.1 & 3.9 & 1.3 \\
\hline & $530^{\mathrm{a}}$ & 59 & 9 & 24100 & 350 & 3.95 & 0.03 & 3.97 & 10.5 & 0.3 & 5.5 & 2.4 \\
\hline & $551^{\mathrm{a}}$ & 220 & 11 & 20200 & 350 & 3.63 & 0.03 & 3.83 & 8.1 & 0.2 & 5.7 & 1.2 \\
\hline & 590 & 119 & 10 & 24050 & 50 & 3.75 & 0.02 & 3.82 & 11.2 & 0.2 & 6.8 & 2.2 \\
\hline & 662 & 67 & 11 & 24300 & 650 & 3.13 & 0.05 & 3.17 & 20.0 & 1.7 & 19.2 & 1.4 \\
\hline & 678 & 100 & 15 & 22950 & 50 & 3.60 & 0.02 & 3.67 & 11.3 & 0.1 & 8.1 & 1.6 \\
\hline \multirow{25}{*}{$\vec{\perp}$} & 692 & 176 & 16 & 25400 & 400 & 3.38 & 0.03 & 3.55 & 15.0 & 0.6 & 10.7 & 2.1 \\
\hline & $731^{\mathrm{a}}$ & 165 & 11 & 21600 & 250 & 3.94 & 0.02 & 4.05 & 8.2 & 0.1 & 4.5 & 1.8 \\
\hline & 748 & 251 & 35 & 10900 & 78 & 3.61 & 0.03 & 3.89 & 3.1 & 0.1 & 3.3 & 0.2 \\
\hline & 768 & 45 & 6 & 17600 & 200 & 3.48 & 0.02 & 3.51 & 7.7 & 0.1 & 8.1 & 0.4 \\
\hline & 769 & 127 & 8 & 22650 & 50 & 3.75 & 0.02 & 3.84 & 9.9 & 0.1 & 6.3 & 1.8 \\
\hline & 789 & 199 & 16 & 17250 & 50 & 4.00 & 0.02 & 4.15 & 5.4 & 0.1 & 3.2 & 1.1 \\
\hline & 803 & 265 & 19 & 23100 & 150 & 3.49 & 0.03 & 3.75 & 10.9 & 0.1 & 7.3 & 1.8 \\
\hline & $843^{\mathrm{a}}$ & 113 & 7 & 21800 & 100 & 3.40 & 0.02 & 3.50 & 11.4 & 0.1 & 9.9 & 0.8 \\
\hline & 859 & 221 & 9 & 22000 & 400 & 3.73 & 0.02 & 3.91 & 8.9 & 0.2 & 5.5 & 1.7 \\
\hline & 864 & 150 & 45 & 22800 & 400 & 3.73 & 0.02 & 3.84 & 10.1 & 0.4 & 6.3 & 1.9 \\
\hline & 922 & 270 & 14 & 25300 & 300 & 3.34 & 0.02 & 3.62 & 14.2 & 0.3 & 9.6 & 2.3 \\
\hline & 1001 & 61 & 4 & 22900 & 100 & 3.60 & 0.02 & 3.63 & 11.5 & 0.1 & 8.6 & 1.4 \\
\hline & $1067^{\mathrm{a}}$ & 63 & 8 & 21800 & 300 & 3.72 & 0.02 & 3.75 & 9.6 & 0.3 & 6.8 & 1.4 \\
\hline & 1078 & 208 & 24 & 24950 & 50 & 3.50 & 0.02 & 3.69 & 13.1 & 0.1 & 8.6 & 2.2 \\
\hline & 1085 & 88 & 5 & 22200 & 100 & 3.50 & 0.02 & 3.57 & 11.3 & 0.1 & 9.2 & 1.1 \\
\hline & 1132 & 103 & 7 & 24300 & 450 & 3.18 & 0.02 & 3.27 & 17.9 & 0.8 & 16.2 & 0.9 \\
\hline & 1141 & 233 & 14 & 20700 & 150 & 3.20 & 0.02 & 3.49 & 10.4 & 0.1 & 9.6 & 0.4 \\
\hline & 1174 & 65 & 9 & 19300 & 200 & 3.93 & 0.02 & 3.96 & 6.9 & 0.1 & 4.5 & 1.2 \\
\hline & 1181 & 71 & 6 & 17300 & 200 & 3.97 & 0.03 & 4.01 & 5.9 & 0.1 & 4.0 & 1.0 \\
\hline & 1364 & 235 & 12 & 20900 & 150 & 3.40 & 0.02 & 3.66 & 9.4 & 0.1 & 7.5 & 0.9 \\
\hline & 1387 & 79 & 4 & 20500 & 100 & 3.85 & 0.02 & 3.90 & 8.1 & 0.1 & 5.3 & 1.4 \\
\hline & 1391 & 161 & 9 & 22300 & 100 & 3.55 & 0.02 & 3.69 & 10.6 & 0.1 & 7.7 & 1.4 \\
\hline & 1482 & 101 & 59 & 10740 & 72 & 3.47 & 0.03 & 3.61 & 3.5 & 0.0 & 4.9 & 0.9 \\
\hline & $1516^{\mathrm{a}}$ & 152 & 17 & 25400 & 650 & 4.24 & 0.05 & 4.29 & 9.9 & 0.5 & 3.7 & 0.2 \\
\hline & 1548 & 195 & 14 & 13300 & 171 & 4.12 & 0.04 & 4.23 & 3.5 & 0.1 & 2.4 & 0.6 \\
\hline
\end{tabular}


TABLE 2-Continued

\begin{tabular}{|c|c|c|c|c|c|c|c|c|c|c|c|}
\hline $\begin{array}{c}\text { WEBDA } \\
\text { ID } \\
(1)\end{array}$ & $\begin{array}{c}V \sin i \\
\left(\mathbf{k m} \mathbf{s}^{-1}\right) \\
(2)\end{array}$ & $\begin{array}{c}\Delta V \sin i \\
\left(\mathbf{k m} \mathbf{s}^{-1}\right) \\
(3)\end{array}$ & $\begin{array}{l}T_{\text {eff }} \\
(\mathrm{K}) \\
(4)\end{array}$ & $\begin{array}{l}\Delta T_{\text {eff }} \\
(\mathrm{K}) \\
(5)\end{array}$ & $\begin{array}{c}\log g \\
(\text { dex }) \\
(6)\end{array}$ & $\begin{array}{c}\Delta \log g \\
(\text { dex }) \\
\quad(7)\end{array}$ & $\begin{array}{c}\log g_{\text {polar }} \\
\text { (dex) } \\
(8)\end{array}$ & $\begin{array}{c}M_{\star} \\
\left(M_{\odot}\right) \\
(9)\end{array}$ & $\begin{array}{c}\Delta M_{\star} \\
\left(M_{\odot}\right) \\
(10)\end{array}$ & $\begin{array}{c}R_{\star} \\
\left(R_{\odot}\right) \\
(11)\end{array}$ & $\begin{array}{c}\Delta R_{\star} \\
\left(R_{\odot}\right) \\
(12)\end{array}$ \\
\hline \multicolumn{12}{|l|}{ NGC 884} \\
\hline 1770 & 232 & 7 & 17650 & 50 & 3.75 & 0.02 & 3.97 & 6.2 & 0.1 & 4.3 & 1.0 \\
\hline 1793 & 136 & 30 & - & - & - & - & - & - & - & - & - \\
\hline $1873^{\mathrm{a}}$ & 166 & 8 & 21600 & 300 & 3.83 & 0.02 & 3.95 & 8.5 & 0.2 & 5.1 & 1.7 \\
\hline 1899 & 137 & 7 & 23500 & 700 & 3.16 & 0.05 & 3.31 & 15.9 & 1.3 & 14.6 & 0.6 \\
\hline $2014^{\mathrm{a}}$ & 293 & 8 & 19000 & 250 & 3.64 & 0.03 & 3.93 & 6.9 & 0.1 & 4.7 & 1.1 \\
\hline 2048 & 100 & 6 & 19400 & 250 & 3.92 & 0.02 & 3.98 & 6.9 & 0.1 & 4.4 & 1.2 \\
\hline 2049 & 413 & 28 & 18050 & 50 & 2.75 & 0.02 & 3.61 & 7.5 & 0.1 & 7.1 & 0.2 \\
\hline 2057 & 151 & 10 & 19450 & 50 & 3.80 & 0.02 & 3.92 & 7.2 & 0.1 & 4.9 & 1.1 \\
\hline 2085 & 284 & 9 & 19000 & 250 & 3.54 & 0.02 & 3.85 & 7.1 & 0.2 & 5.3 & 0.9 \\
\hline 2086 & 146 & 34 & - & - & - & - & - & - & - & - & - \\
\hline 2094 & 80 & 3 & 20350 & 50 & 4.00 & 0.02 & 4.04 & 7.4 & 0.1 & 4.3 & 1.6 \\
\hline 2112 & 89 & 8 & 22300 & 150 & 3.80 & 0.02 & 3.85 & 9.4 & 0.2 & 6.0 & 1.7 \\
\hline 2119 & 50 & 5 & 22400 & 100 & 3.95 & 0.02 & 3.97 & 8.9 & 0.1 & 5.1 & 1.9 \\
\hline 2185 & 202 & 12 & 17000 & 200 & 3.93 & 0.02 & 4.10 & 5.5 & 0.1 & 3.5 & 1.0 \\
\hline 2190 & 335 & 28 & 20400 & 450 & 3.18 & 0.02 & 3.67 & 8.9 & 0.3 & 7.2 & 0.8 \\
\hline $2191^{\mathrm{c}}$ & 263 & 16 & 18050 & 50 & 3.80 & 0.02 & 4.04 & 6.2 & 0.1 & 3.9 & 1.1 \\
\hline 2218 & 248 & 35 & 10110 & 57 & 3.84 & 0.03 & 4.07 & 2.6 & 0.1 & 2.5 & 0.1 \\
\hline 2227 & 62 & 7 & 23600 & 1300 & 3.00 & 0.10 & 3.07 & 21.0 & 4.0 & 22.2 & 4.8 \\
\hline $2232^{\mathrm{c}}$ & 115 & 5 & 22150 & 50 & 3.90 & 0.02 & 3.97 & 8.8 & 0.1 & 5.1 & 1.8 \\
\hline 2255 & 333 & 15 & 20200 & 150 & 3.38 & 0.02 & 3.81 & 8.2 & 0.1 & 5.9 & 1.1 \\
\hline $2311^{\mathrm{b}}$ & 51 & 5 & 23400 & 400 & 3.48 & 0.02 & 3.50 & 13.3 & 0.5 & 10.8 & 1.3 \\
\hline $2336^{\mathrm{a}}$ & 173 & 9 & 19200 & 200 & 3.92 & 0.02 & 4.05 & 6.7 & 0.1 & 4.0 & 1.3 \\
\hline 2347 & 84 & 11 & 21800 & 100 & 4.00 & 0.02 & 4.04 & 8.3 & 0.1 & 4.6 & 1.9 \\
\hline 2361 & 68 & 8 & 25500 & 500 & 3.37 & 0.05 & 3.40 & 17.7 & 0.8 & 13.9 & 1.9 \\
\hline 2392 & 87 & 17 & 21200 & 150 & 3.75 & 0.02 & 3.81 & 8.8 & 0.1 & 6.1 & 1.3 \\
\hline 2520 & 45 & 10 & 23200 & 350 & 3.67 & 0.02 & 3.68 & 11.4 & 0.3 & 8.0 & 1.7 \\
\hline 2540 & 271 & 23 & 19800 & 250 & 3.53 & 0.02 & 3.82 & 7.9 & 0.2 & 5.7 & 1.1 \\
\hline 2555 & 129 & 6 & 18250 & 50 & 4.05 & 0.02 & 4.13 & 6.0 & 0.1 & 3.5 & 1.3 \\
\hline 2605 & 54 & 27 & 24600 & 450 & 3.58 & 0.02 & 3.60 & 13.6 & 0.5 & 9.7 & 1.9 \\
\hline 2622 & 373 & 35 & 17200 & 200 & 3.38 & 0.03 & 3.91 & 6.1 & 0.1 & 4.6 & 0.8 \\
\hline $2716^{\mathrm{a}}$ & 198 & 7 & 19650 & 50 & 3.75 & 0.02 & 3.92 & 7.3 & 0.1 & 4.9 & $\begin{array}{l}1.2 \\
1.2\end{array}$ \\
\hline 2729 & 208 & 17 & 10410 & 74 & 3.83 & 0.03 & 4.01 & 2.8 & 0.1 & 2.7 & 0.1 \\
\hline 2907 & 233 & 52 & 13700 & 150 & 3.86 & 0.03 & 4.07 & 3.9 & 0.1 & 3.0 & 0.4 \\
\hline
\end{tabular}

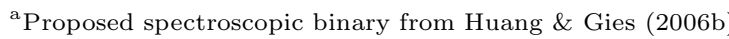

${ }^{\mathrm{b}}$ Eclipsing binary from Southworth et al. (2004)

${ }^{\mathrm{c}}$ Proposed eclipsing binary from Saesen et al. (2010) 
TABLE 3

Physical Parameters of Be Star Cluster Members

\begin{tabular}{|c|c|c|c|c|c|c|c|c|c|c|c|c|}
\hline $\begin{array}{c}\text { WEBDA } \\
\text { ID } \\
(1)\end{array}$ & $\begin{array}{c}V \sin i \\
\left(\mathbf{k m} \mathbf{s}^{-1}\right) \\
(2)\end{array}$ & $\begin{array}{c}\Delta V \sin i \\
\left(\mathbf{k m} \mathbf{s}^{-1}\right) \\
(3)\end{array}$ & $\begin{array}{l}T_{\text {eff }} \\
(\mathbf{K}) \\
(4)\end{array}$ & $\begin{array}{c}\Delta T_{\text {eff }} \\
(\mathbf{K}) \\
(5)\end{array}$ & $\begin{array}{c}\log g \\
(\text { dex }) \\
(6)\end{array}$ & $\begin{array}{c}\Delta \log g \\
(\text { dex }) \\
(7)\end{array}$ & $\begin{array}{c}\log g_{\text {polar }} \\
\text { (dex) } \\
(8)\end{array}$ & $\begin{array}{c}M_{\star} \\
\left(M_{\odot}\right) \\
(9)\end{array}$ & $\begin{array}{c}\Delta M_{\star} \\
\left(M_{\odot}\right) \\
(10)\end{array}$ & $\begin{array}{c}R_{\star} \\
\left(R_{\odot}\right) \\
(11)\end{array}$ & $\begin{array}{c}\Delta R_{\star} \\
\left(R_{\odot}\right) \\
(12)\end{array}$ & $\begin{array}{c}\text { Notes } \\
(13)\end{array}$ \\
\hline \multicolumn{13}{|l|}{ NGC 869} \\
\hline 49 & 172 & 21 & 25400 & 1790 & 3.54 & 0.23 & 3.54 & 15.4 & 2.5 & 11.0 & 2.0 & $\begin{array}{l}\text { No Emission Observed(1); } \\
\text { Emission Observed }(2,7.8)\end{array}$ \\
\hline 146 & 195 & 6 & 25600 & 600 & 3.66 & 0.05 & 3.80 & 12.7 & 0.7 & 7.4 & 2.6 & $\begin{array}{l}\text { No Emission Observed }(2,8) ; \\
\text { Emission Observed }(4)\end{array}$ \\
\hline 309 & - & - & 27700 & 1790 & 3.74 & 0.23 & 3.74 & 15.8 & 1.6 & 8.8 & 2.1 & Emission Observed $(1,2,4,5,8)$ \\
\hline 517 & 178 & 13 & - & - & - & - & - & - & - & - & - & Emission Observed $(4,6,7,8)$ \\
\hline $566^{\mathrm{a}}$ & 306 & 25 & 22100 & 100 & 3.25 & 0.02 & 3.63 & 10.8 & 0.1 & 8.4 & 1.2 & $\begin{array}{l}\text { No Emission Observed }(1,4,8) \text {; } \\
\text { Emission Observed }(2,7,8)\end{array}$ \\
\hline $717^{\mathrm{b}}$ & 126 & 22 & 26700 & 650 & 3.91 & 0.05 & 3.96 & 12.5 & 0.8 & 6.1 & 3.2 & $\begin{array}{l}\text { No Emission Observed }(6,8) \\
\text { Emission Observed(3) }\end{array}$ \\
\hline 846 & 205 & 19 & - & - & - & - & - & - & - & - & - & Emission Observed $(4,6,7,8)$ \\
\hline 847 & 87 & 11 & 23000 & 1790 & 3.41 & 0.24 & 3.41 & 13.7 & 0.2 & 12.1 & 2.8 & Emission Observed $(2,4,6,7,8)$ \\
\hline 992 & - & - & 21700 & 1790 & 3.60 & 0.24 & 3.60 & 10.7 & 0.1 & 8.6 & 2.0 & Emission Observed $(6,8)$ \\
\hline 1057 & - & - & 21300 & 1790 & 2.69 & 0.25 & 2.69 & 29.5 & 0.2 & 40.8 & 9.6 & Emission Observed $(6,8)$ \\
\hline 1161 & - & - & 23400 & 1790 & 3.77 & 0.24 & 3.77 & 11.1 & 0.2 & 7.2 & 1.7 & Emission Observed $(2,4,5,6,7,8)$ \\
\hline 1261 & 285 & 78 & 26400 & 1790 & 3.72 & 0.23 & 3.72 & 14.3 & 0.6 & 8.6 & 2.0 & Emission Observed $(2,4,5,6,7,8)$ \\
\hline 1268 & 151 & 12 & 25400 & 500 & 3.46 & 0.05 & 3.59 & 14.6 & 0.6 & 10.1 & 2.2 & $\begin{array}{l}\text { No Emission Observed }(6,8) ; \\
\text { Emission Observed }(4)\end{array}$ \\
\hline 1278 & 197 & 12 & 25100 & 1790 & 4.24 & 0.24 & 4.24 & 9.9 & 0.5 & 3.9 & 0.9 & Emission Observed $(4,6,7,8)$ \\
\hline 1282 & - & - & 22700 & 1790 & 3.95 & 0.24 & 3.95 & 9.2 & 0.2 & 5.3 & 1.2 & Emission Observed $(2,4,5,6,7,8)$ \\
\hline \multicolumn{13}{|l|}{ NGC 884} \\
\hline $1702^{\mathrm{a}}$ & - & - & 24400 & 1790 & 3.67 & 0.24 & 3.67 & 12.6 & 0.3 & 8.6 & 2.0 & Emission Observed $(1,2,4,6,7,8)$ \\
\hline 1772 & 379 & 28 & - & - & - & - & - & . & - & - & - & $\begin{array}{l}\text { No Emission Observed(4); } \\
\text { Emission Observed }(6,8)\end{array}$ \\
\hline $1926^{\mathrm{a}}$ & 106 & 17 & 27400 & 1790 & 3.89 & 0.23 & 3.89 & 13.9 & 1.2 & 7.0 & 1.6 & Emission Observed $(2,4,6,7,8)$ \\
\hline 1977 & - & - & 20400 & 1790 & 4.27 & 0.25 & 4.27 & 6.8 & 0.1 & 3.2 & 0.7 & Emission Observed $(2,4,6,7,8)$ \\
\hline $2088^{\mathrm{a}}$ & - & - & 21900 & 1790 & 3.72 & 0.24 & 3.72 & 10.0 & 0.1 & 7.2 & 1.7 & Emission Observed $(2,4,5,6,7,8)$ \\
\hline $2091^{\mathrm{a}}$ & 236 & 40 & 18800 & 1790 & 3.97 & 0.26 & 3.97 & 6.7 & 0.1 & 4.4 & 1.0 & Emission Observed $(4,6,7,8)$ \\
\hline 2138 & 153 & 37 & 24100 & 1790 & 3.53 & 0.24 & 3.53 & 13.8 & 0.2 & 10.5 & 2.5 & Emission Observed $(1,2,4,5,7,8)$ \\
\hline 2165 & - & - & 24800 & 1790 & 3.79 & 0.24 & 3.79 & 11.9 & 0.3 & 7.3 & 1.7 & Emission Observed $(2,4,5,6,7,8)$ \\
\hline 2262 & 265 & 19 & 24900 & 1400 & 3.51 & 0.08 & 3.75 & 12.3 & 1.4 & 7.7 & 2.2 & $\begin{array}{l}\text { No Emission Observed }(4,8) ; \\
\text { Emission Observed }(2,6)\end{array}$ \\
\hline 2284 & - & - & 26500 & 1790 & 3.77 & 0.23 & 3.76 & 14.0 & 0.7 & 8.1 & 1.9 & Emission Observed $(2,4,5,6,7,8)$ \\
\hline 2468 & 134 & 46 & 10070 & 48 & 3.88 & 0.03 & 4.00 & 2.7 & 0.1 & 2.7 & 0.1 & $\begin{array}{l}\text { No Emission Observed(8); } \\
\text { Emission Observed(7) }\end{array}$ \\
\hline 2563 & 308 & 74 & 26100 & 1790 & 4.02 & 0.23 & 4.02 & 11.6 & 1.5 & 5.5 & 2.7 & Emission Observed $(2,4,6,7,8)$ \\
\hline 2771 & - & - & 21800 & 1790 & 3.99 & 0.24 & 3.99 & 8.5 & 0.1 & 4.9 & 1.1 & Emission Observed $(2,4,7,8)$ \\
\hline
\end{tabular}

Bragg \& Kenvon (2002), (7) Be candidate with observed IR excesses in Currie et al. (2008), (8) This work.

${ }^{\text {a }}$ Proposed spectroscopic binary from Huang \& Gies (2006b)

${ }^{\mathrm{b}}$ Candidate spectroscopic binary from Strom et al. (2005) 